Europhysics Letters

PREPRINT

\title{
Interplay between incommensurate phases in the cuprates
}

\author{
Marcin RacZKowski $^{1}$, Raymond Frésard ${ }^{2}$ and Andrzej M. Oleś $\hat{S}^{1,3}$ \\ 1 Marian Smoluchowski Institute of Physics, Jagellonian University, ul. Reymonta 4, \\ PL-30059 Kraków, Poland \\ 2 Laboratoire CRISMAT, UMR CNRS-ENSICAEN(ISMRA) 6508, 6 Bld. du Maréchal \\ Juin, F-14050 Caen, France \\ 3 Max-Planck-Institut für Festkörperforschung, Heisenbergstrasse 1, D-70569 Stuttgart, \\ Germany
}

PACS. 74.72. -h - Cuprate superconductors (high-Tc and insulating parent compounds).

PACS. 71.45.Lr - Charge-density-wave systems.

PACS. 71.10.Fd - Lattice fermion models (Hubbard model, etc.).

\begin{abstract}
We establish the qualitative behavior of the incommensurability $\epsilon$, optimal domain wall filling $\nu$ and chemical potential $\mu$ for increasing doping by a systematic slaveboson study of an array of vertical stripes separated by up to $d=11$ lattice constants. Our findings obtained in the Hubbard model with the next-nearest neighbor hopping $t^{\prime}=-0.15 t$ agree qualitatively with the experimental data for the cuprates in the doping regime $x \lesssim 1 / 8$. It is found that $t^{\prime}$ modifies the optimal filling $\nu$ and triggers the crossover to the diagonal $(1,1)$ spiral phase at increasing doping, stabilized already at $x \simeq 0.09$ for $t^{\prime}=-0.3 t$.
\end{abstract}

Interest in transition metal oxides has never been restricted to the most spectacular phenomenon of the high- $T_{c}$ superconductivity, but also concerns, inter alia, metal-insulator transitions, colossal magnetoresistance, and orbital ordering [1]. Among these phenomena the so-called stripe phases attract much attention. In this context the recent indications of universal magnetic excitations in doped cuprates are intriguing [2]. Remarkably, some features of the magnetic spectra including their anisotropic two-dimensional character established in a detwinned $\mathrm{YBa}_{2} \mathrm{Cu}_{3} \mathrm{O}_{6+\delta}$ (YBCO) sample [3] can be understood in terms of fluctuating stripes suggesting that they are one of generic properties of the copper oxides [4].

Typically, such states result from the competition between the superexchange interaction, which stabilize the antiferromagnetic (AF) long-range order in the parent Mott insulator, and the kinetic energy of doped holes. Indeed, the magnetic energy is gained when electrons occupy the neighboring sites and their spins coupled by the superexchange order as in the Néel state, whereas the kinetic energy is gained when the holes can move and the AF order is locally suppressed along a domain wall (DW), leading to the formation of site-centered (SC) stripes. Other possible structures are bond-centered (BC) stripes. In this case, AF domains with a lower hole density and a stronger spin polarization are separated by DWs given by ladders with an increased hole density and a weak ferromagnetic order on the rungs.

The most direct evidence for stripe phases in doped antiferromagnets has come from neutron scattering studies in which charge and spin modulations are identified by the appearance

(c) EDP Sciences 
of several extra incommensurate (IC) Bragg peaks [5]. Indeed, neutron diffraction measurements performed on $\mathrm{La}_{1.6-x} \mathrm{Nd}_{0.4} \mathrm{Sr}_{x} \mathrm{CuO}_{4}$ (Nd-LSCO), a model compound for which the evidence of vertical spin and charge stripe order is strongest, revealed that magnetic peaks are displaced from the $\mathrm{AF}$ maximum at $\mathbf{Q}_{\mathrm{AF}}=(\pi, \pi)$ to the points $\mathbf{Q}=\pi(1 \pm 2 \epsilon, 1)$ and $\mathbf{Q}=\pi(1,1 \pm 2 \epsilon)[6]$. Remarkably, the incommensurability $\epsilon$ varies linearly with doping $\epsilon=x$ in the underdoped regime of $x<1 / 8$ meaning a fixed stripe filling $\nu=1 / 2$. A similar value of the number of holes per DW $\nu=0.59$ has also been estimated in a recent resonant soft X-ray scattering study of $\mathrm{La}_{2-x} \mathrm{Ba}_{x} \mathrm{CuO}_{4}$ (LBCO) which is a more direct evidence of charge modulation [7]. In contrast, beyond $x=1 / 8$, one finds in experiment a lock-in effect with $\epsilon=1 / 8$, corresponding to a robust stripe phase with a charge (magnetic) unit cell consisting of four (eight) sites, and the AF domains with three atoms along the $x$ direction. The essentially identical modulation and doping dependence of $\epsilon$ was observed in superconducting crystals of $\mathrm{La}_{2-x} \mathrm{Sr}_{x} \mathrm{CuO}_{4}$ (LSCO) with $x>0.05$ [8]. Also in YBCO the incommensurability increases first linearly and next saturates at the effective hole doping $x \simeq 0.10[9]$.

Apart from the neutron scattering, stripe phases have also measurable consequences in angle-resolved photoemission spectroscopy (ARPES) [10]. Indeed, the low-energy spectral weight of Nd-LSCO at $x=1 / 8$, is mostly concentrated along the antinodal $\Gamma-X$ and $\Gamma-Y$ directions, while there is a distinct gap for charge excitations around the $S=(\pi / 2, \pi / 2)$ point, as expected in the SC stripe picture [11-13]. In contrast, ARPES spectra of both LSCO and Nd-LSCO at $x=0.15$ have revealed the existence of appreciable spectral weight along the nodal $\Gamma-S$ direction. Therefore, as the BC stripes reproduce quite well the nodal segments [12], it seems that upon increasing doping they are formed at the expense of the $\mathrm{SC}$ ones. The relevance of the $\mathrm{BC}$ stripes at the doping level $x=0.15$ is also supported by recent studies which have yielded pronounced spectral weight both in the nodal and antinodal directions, reproducing quite well the experimental results in Nd-LSCO and LSCO [14].

An alternative scenario which might explain the IC spin structure is a deformation of the AF order which optimizes a hole motion within a spiral phase [15]. The interest in the spiral state was recently renewed due to experimental results indicating a spin glass behavior of LSCO at small doping consistent with this scenario [16]. On the one hand, observation of the charge order in Nd-LSCO and LBCO rules out the spiral phase since the density of holes is then expected to be uniform. On the other hand, the question whether the charge order is a generic feature of the cuprates is far from being resolved yet [5]. In fact, Lindgård [17] has shown that spiral states can also resolve the universality of magnetic excitations in the cuprates and provide a competing paradigm with the stripe phase concept.

Hence, an interplay between domain wall structures and spiral phases poses an interesting problem to study [18] even though the searching for the optimal filling of domain walls corresponding to the true ground state is rather a formidable task. Therefore, we limit ourselves to vertical site-centered (VSC) and vertical bond-centered (VBC) stripe phases, with the size of the AF domains varying from $d=11$ to 3 lattice constants. In both structures, the largest distance $d=11$ corresponds to a unit cell with 22 atoms. Note that the same length of the magnetic unit cell for a fixed $d$ makes the $\mathrm{SC}$ and $\mathrm{BC}$ structures practically indistinguishable from each other in neutron diffraction experiments.

We study the stripe and spiral phases using the Hubbard model,

$$
H=-t \sum_{\langle i j\rangle \sigma}\left(c_{i \sigma}^{\dagger} c_{j \sigma}+H . c .\right)-t^{\prime} \sum_{\langle\langle i j\rangle\rangle \sigma}\left(c_{i \sigma}^{\dagger} c_{j \sigma}+H . c .\right)+U \sum_{i} n_{i \uparrow} n_{i \downarrow},
$$

where the electron hopping $t$ involves the nearest neighbor sites $\langle i j\rangle, t^{\prime}$ the next-nearest neighbor sites $\langle\langle i j\rangle\rangle$, and $U$ stands for the on-site Coulomb interaction. There are several 

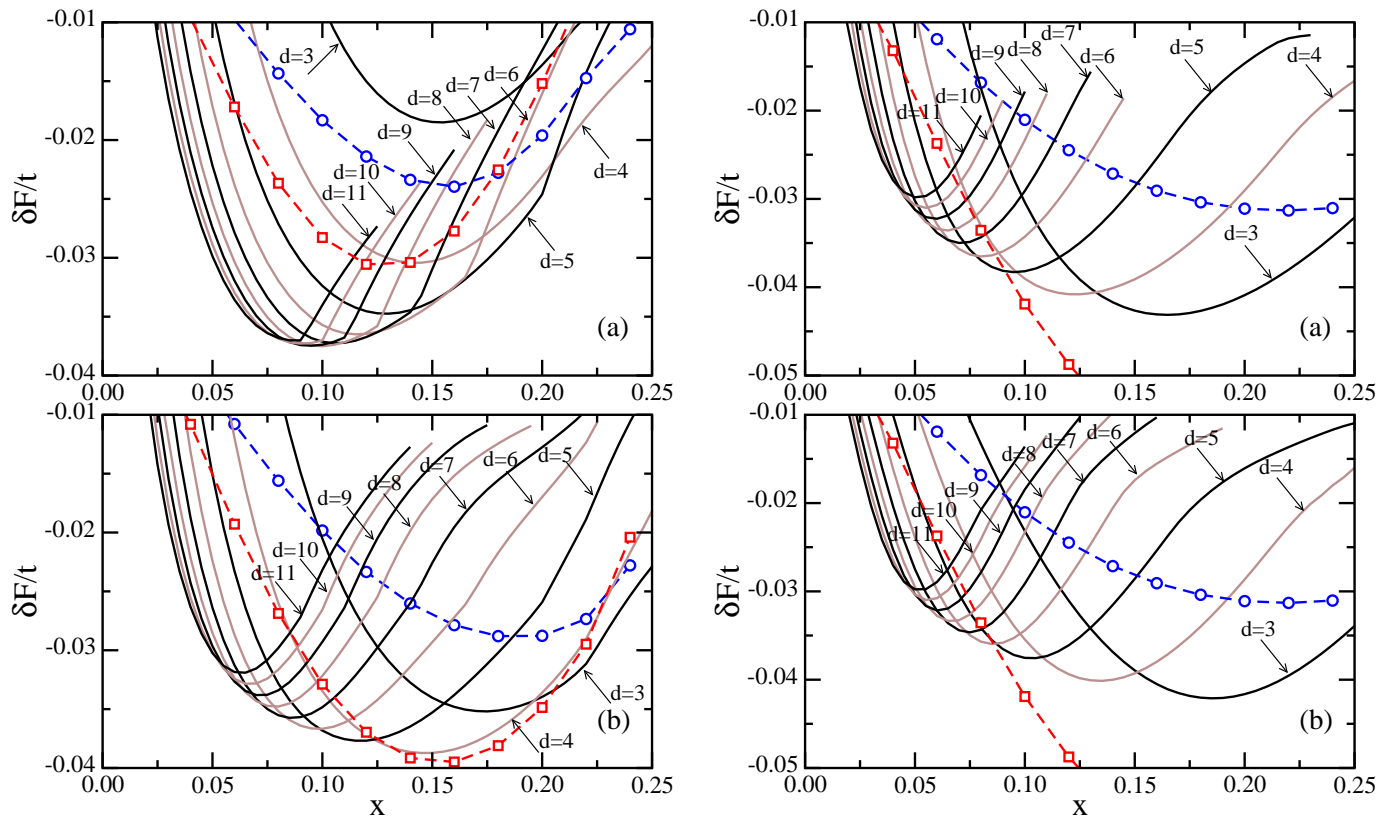

Fig. 1

Fig. 2

Fig. 1 - (Color online) Free energy gain $\delta F$ per site in the VSC stripe phases with respect to the AF phase as a function of doping $x$, as obtained for the $t-t^{\prime}-U$ model (1) with $U=12 t$ and: (a) $t^{\prime}=0$; (b) $t^{\prime}=-0.15 t$. Domain walls are separated by $d=3, \ldots, 11$ lattice constants. Circles and squares show the corresponding data for $(1,0)$ and $(1,1)$ spiral order, respectively.

Fig. 2 - (Color online) Free energy gain $\delta F$ per site as in fig. 1 obtained for the $t-t^{\prime}-U$ model $(1)$ with $U=12 t$ and $t^{\prime}=-0.3 t$ for: (a) VSC stripe phases; (b) VBC stripe phases.

experimental and theoretical studies suggesting that $t^{\prime}$ is finite in the cuprates. For example, topology of the Fermi surface seen by ARPES [10] can be only understood by introducing $t^{\prime}$ [19]. It also offers an explanation for the variation of $T_{c}$ among different families of holedoped cuprates [20]. Moreover, exact diagonalization (ED) studies have shown that while the $d$-wave superconductivity correlation is slightly suppressed by $t^{\prime}<0$ in the underdoped region, it is substantially enhanced in the optimally doped and overdoped regions, indicating that $t^{\prime}$ is of great importance for the pairing instability [21].

We employ a rotationally invariant version of the slave-boson (SB) approach in spin space [22], which opens a possibility of studying both noncanted stripe phases and spiral order on equal footing. More details on the calculation method can be found in ref. [23]. Calculations were carried out on square clusters with the linear dimension along the $x$ direction (perpendicular to the DWs) chosen as an even multiplicity of the elementary stripe unit cell dimension in all cases, so that the cluster size used for the considered unit cells varied from $128 \times 128$ up to $144 \times 144$. These calculations became possible by developing an efficient scheme in the reciprocal space which makes use of the stripe symmetry [24]. For so large systems the finite size effects are below $10^{-5} t$ and one is able to obtain a realistic comparison of the free energies of structures with different size of the unit cell. In the numerical studies, we have chosen $U=12 t$, which gives the ratio of $J / t=1 / 3$ (with the superexchange $J=4 t^{2} / U$ ), being a value representative for LSCO [25]. 
EUROPHYSICS LETTERS

We begin with the free energy gain of the VSC stripe phase with respect to the AF phase, $\delta F \equiv F-F_{\mathrm{AF}}$, shown in fig. 1 as a function of doping $x$ for representative values of the next-nearest neighbor hopping, i.e., $t^{\prime}=0, t^{\prime}=-0.15 t$ (VBC phases are addressed below). Quite generally, one observes that the energy favors the stripe phases with the largest distance $d=11$ between DWs for the lowest values of $x$, and the curves for next smaller $d-1$ systematically cross the ones for $d$ upon increasing doping, meaning that stripe phases with gradually decreasing AF domains become optimal phases. For $t^{\prime}=0$, this effect continues until the $d=4$ stripe phase is reached as the $d=3$ stripe phase is a highly-excited state in this case. On the contrary, finite $t^{\prime}$ results in a deeper energy minimum of the $d=3$ phase and stabilizes it in the overdoped regime $x \geq 0.2$. Next, except for the smallest $d \leq 4$ cases, increasing $\left|t^{\prime}\right|$ shifts the free energy minima towards lower doping level which should affect the filling of the DWs and the charge distribution in the stripe ground state. Remarkably, for $t^{\prime}=-0.15 t$, a value very close to that derived for LSCO [26], one finds that the most stable stripes are separated by $d=4$ lattice spacings in a sizeable doping range above $x \simeq 1 / 8$, both for the $\mathrm{SC}$ and $\mathrm{BC}$ stripe phase, in agreement with the neutron scattering experiment [8] and with dynamical mean field theory (DMFT) calculations [13] for LSCO.

Regarding the spiral phases, upon hole doping away from half-filling the AF order becomes immediately unstable towards a diagonal $(1,1)$ spiral order with $\mathbf{Q}=\pi(1-2 \epsilon, 1-2 \epsilon)$ and then at higher doping $x \simeq 0.18$ towards a vertical $(1,0)$ one characterized by $\mathbf{Q}=\pi(1-2 \epsilon, 1)$, as suggested by early SB studies of the Hubbard model [27]. However, a finite next-nearest neighbor $t^{\prime}=-0.15 t$ has severe consequences for the interplay between both spiral phases and shifts the crossover towards a higher doping $x \simeq 0.23$ in agreement with the SB studies of the $t-t^{\prime}-J$ model [28]. It also affects the competition between the spiral and stripe phases so that at $x>1 / 8$ the latter become unstable with respect to the $(1,1)$ spirals. However, the energy difference between the most stable stripe phase with $d=4$ and the lowest energy spiral phase is less than $0.002 t$, which is considered to be too small to lead to an unambiguous conclusion. Indeed, comparison of the SB data with available ED results on a $4 \times 4$ cluster obtained in the Hubbard model at $t^{\prime}=0$ and $x=1 / 8$ doping [29] shows that taking into account stripe phases allows one to approach closer the ED energy than the best spiral phase but due to the quantum fluctuations the energy difference remains at the $9 \%$ level ( $c f$. table I).

In contrast, a larger value of the ratio $\left|t^{\prime} / t\right|=0.3$ as expected for YBCO [26], clearly drives the system towards the diagonal $(1,1)$ spiral phase and the crossover from the VSC (VBC) stripe phase appears already slightly below (above) $x=0.09$, respectively, as depicted in fig. 2. Note that in the case of the SC DWs it is the $d=4$ stripe phase which is unstable towards the spiral order and indeed the smallest distance between DWs established in YBCO corresponds to $d=5$ [9]. Our results are then consistent with both density matrix renormalization group [30] and Hartree-Fock [31] studies indicating the suppression of the stripe phases with increasing $\left|t^{\prime}\right|$. Moreover, large $\left|t^{\prime}\right|$ removes also the degeneracy between the two stripe structures which shows up at $t^{\prime}=-0.15 t$. Indeed, as reported in table $\amalg$ the energy difference between the lowest energy $\mathrm{SC}$ and $\mathrm{BC}$ configuration in the doping regime $x<0.2$ is

TABLE I - Comparison of the free energy $F$ per site for various phases as found within the $S B$ approximation in the Hubbard model at $x=1 / 8$ with the ED data of ref. [29]. The ground state (VBC and VSC) stripe phases are separated by $d=7$ lattice constants. Parameters: $U=12 t, t^{\prime}=0$.

\begin{tabular}{ccccccc}
\hline phase & AF & $(1,0)$ & $(1,1)$ & VBC & VSC & ED [29] \\
\hline$F / t$ & -0.5393 & -0.5613 & -0.5700 & -0.5756 & -0.5756 & -0.6282 \\
\hline
\end{tabular}


comparable to the accuracy of the present calculations. In contrast, when $t^{\prime}=-0.3 t$, the SC stripe phase is noticeably more stable than its $\mathrm{BC}$ counterpart even for $x<0.2$. The crossover towards the $\mathrm{BC}$ stripes above $x=0.2$ remains in agreement with a recent analysis of the propagation of a hole inside the $\mathrm{BC}$ DW which has shown that such a DW structure enables a larger kinetic energy gain than a narrower SC one, which becomes especially important at a large doping level when the distances between stripes are small [32].

The experimental data should be compared with our findings concerning the doping dependence of the magnetic incommensurability $\epsilon=1 / 2 d$ and the optimal stripe filling, $\nu=N_{\mathrm{h}} /\left(N_{y} N_{\mathrm{DW}}\right)$, where $N_{\mathrm{h}}$ is the hole excess compared to half-filling, whereas $N_{y}$ stands for the actual length of the cluster with $N_{\mathrm{DW}}$ DWs along the $y$ direction. The ground state properties of both SC and BC phases are shown in fig. 3. The points in fig. 3 were deduced from figs. [1 and 2 and correspond to the middle of the stability region of the lowest energy configuration. The only exception is the $d=3$ case with $t^{\prime}=-0.3 t$ in which they are plotted for the minimum of the free energy. Such a choice guarantees that, at each particular doping level, a considered stripe phase with a given periodicity $d$ would be indeed realized at least in the vast majority of the system. Comparing fig. [3 with the experimental results in LSCO $[7,8]$, one finds that the robust stability of the half-filled $(\nu=1 / 2) d=4$ stripes at $x=1 / 8$ requires, in agreement with the previous SB studies on small $16 \times 16$ clusters [33], a finite next-nearest neighbor hopping $t^{\prime}<-0.15 t$. In fact, for $t^{\prime}=-0.15 t$ present calculations give almost a linear dependence $\epsilon=x$ for $x \lesssim 1 / 8$ and may be considered as reproducing a lock-in effect in a sizeable doping range above $x \simeq 1 / 8$ until the $d=3$ stripe phase sets in. We emphasize that stable $d=3$ stripe phases with $\epsilon=1 / 6$ have also been found in the SB studies of the three-band Hubbard model in the doping regime $x>0.225$ [34]. The apparent absence of this phase in the experimental data for LSCO could follow from two effects which go beyond the present study: $(i)$ repulsion between DWs, and (ii) quantum fluctuations which destabilize the ladder-like AF domains.

Remarkably, in the regime where $\epsilon$ follows linearly $x$, an increasing density of stripes allows

TABLE II - Comparison of the ground state free energy $F$ per site for the VSC and VBC stripe phases as found in the $t-t^{\prime}-U$ model with $U=12 t$ and: $t^{\prime}=-0.15 t$ and $t^{\prime}=-0.3 t$.

\begin{tabular}{ccccccccc}
\hline & \multicolumn{4}{c}{$t^{\prime}=-0.15 t$} & \multicolumn{3}{c}{$t^{\prime}=-0.3 t$} \\
& & $\mathrm{VSC}$ & \multicolumn{2}{c}{$\mathrm{VBC}$} & & $\mathrm{VSC}$ & \multicolumn{2}{c}{$\mathrm{VBC}$} \\
\hline$x$ & $d$ & $F / t$ & $d$ & $F / t$ & $d$ & $F / t$ & $d$ & $F / t$ \\
\hline 0.050 & 11 & -0.4263 & 11 & -0.4263 & 9 & -0.4289 & 10 & -0.4280 \\
0.055 & 10 & -0.4360 & 10 & -0.4359 & 8 & -0.4388 & 9 & -0.4378 \\
0.060 & 9 & -0.4456 & 9 & -0.4455 & 7 & -0.4488 & 8 & -0.4477 \\
0.070 & 8 & -0.4649 & 8 & -0.4648 & 6 & -0.4686 & 7 & -0.4673 \\
0.080 & 7 & -0.4841 & 7 & -0.4840 & 5 & -0.4882 & 6 & -0.4869 \\
0.090 & 6 & -0.5034 & 6 & -0.5032 & 5 & -0.5080 & 5 & -0.5064 \\
0.100 & 5 & -0.5225 & 6 & -0.5224 & 4 & -0.5275 & 5 & -0.5260 \\
0.120 & 5 & -0.5607 & 5 & -0.5607 & 4 & -0.5659 & 4 & -0.5645 \\
0.140 & 4 & -0.5985 & 4 & -0.5983 & 3 & -0.6022 & 4 & -0.6005 \\
0.160 & 4 & -0.6342 & 4 & -0.6342 & 3 & -0.6370 & 3 & -0.6344 \\
0.180 & 4 & -0.6671 & 4 & -0.6670 & 3 & -0.6678 & 3 & -0.6672 \\
0.200 & 3 & -0.6978 & 3 & -0.6983 & 3 & -0.6949 & 3 & -0.6956 \\
0.250 & 3 & -0.7682 & 3 & -0.7689 & 3 & -0.7475 & 3 & -0.7493 \\
0.300 & 3 & -0.8242 & 3 & -0.8245 & 3 & -0.7822 & 3 & -0.7833 \\
\hline
\end{tabular}



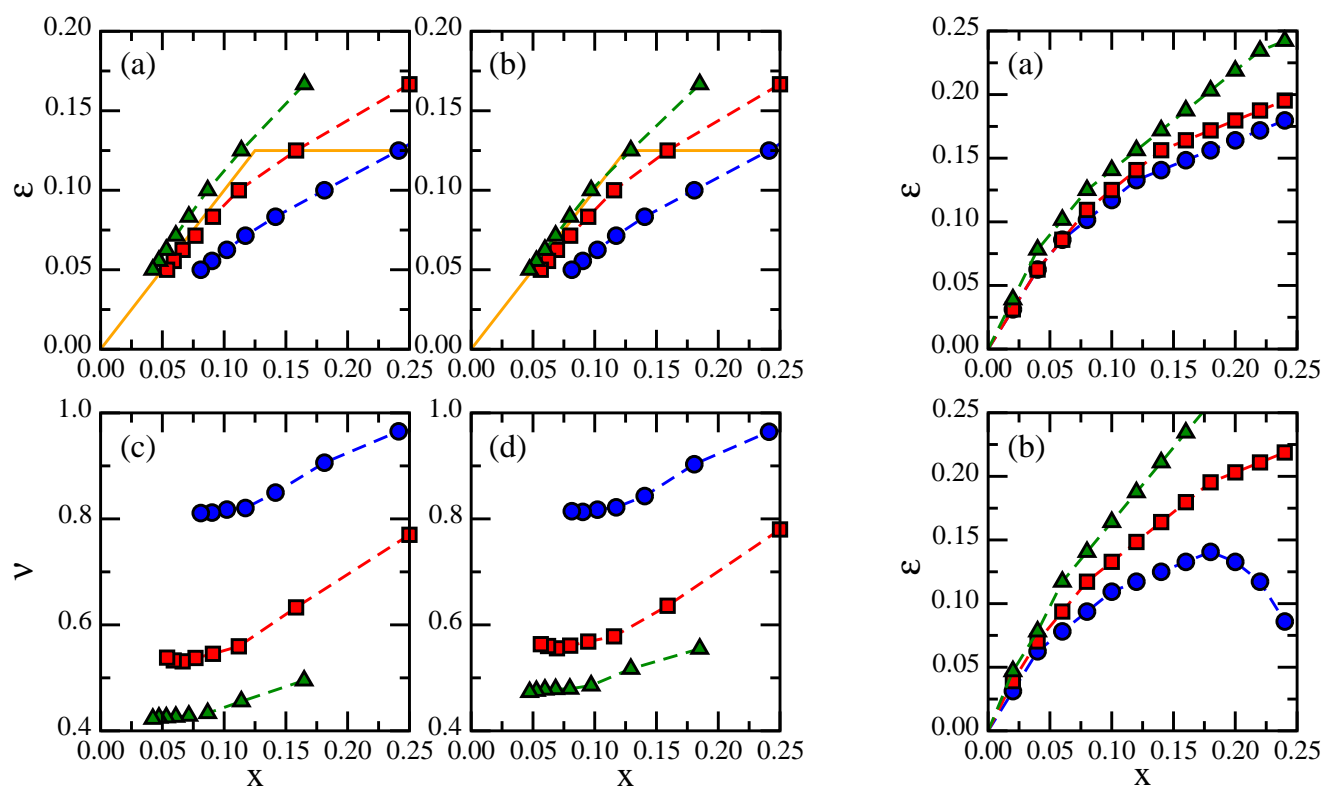

Fig. 3

Fig. 4

Fig. 3 - (Color online) Doping dependence of: (a,b) the magnetic incommensurability $\epsilon$, and (c,d) stripe filling $\nu$ for the VSC (left) as well as VBC (right) stripe ground state deduced from figs. 11 and 2 for $t^{\prime}=0$ (circles), $t^{\prime}=-0.15 t$ (squares), and $t^{\prime}=-0.3 t$ (triangles). Solid line in (a,b) shows the idealized experimental behavior of $\epsilon$ in LSCO [8].

Fig. 4 - (Color online) Doping dependence of the magnetic incommensurability $\epsilon$ as obtained at $U=12 t$ for: (a) $(1,0)$ and (b) $(1,1)$ spiral phase. Meaning of symbols as in fig. 3

the system to maintain fixed filling $\nu$, but its actual value strongly depends on $t^{\prime}$. This, in turn, results in almost doping independent chemical potential $\mu$ explaining the experimentally observed pinning of $\mu$ [35]. In contrast, in the overdoped region with a lock-in effect of $\epsilon$, the size of the AF domains saturates, doped holes penetrate into the AF domains, and $\mu$ varies fast with doping. Unfortunately, for $t^{\prime}=-0.15 t$, the established shift of $\mu$ exceeds the experimental value by a factor close to 2 . Therefore, the present effective model can only explain qualitative trends and one needs to carry out calculations within more realistic multiband models including oxygen orbitals in order to reproduce quantitatively the experimental data.

Finite $t^{\prime}<0$ also modifies the spiral order - here one finds that the deviation of the spiral wavevector $\mathbf{Q}$ from the AF one strongly accelerates, as shown in fig. 4. It is worth noticing that the increase of the incommensurability with increasing $\left|t^{\prime}\right|$ is consistent both with quantum Monte Carlo [36] and with DMFT [37] results.

Summarizing, we have performed systematic studies of incommensurate phases by considering variable size of AF domains in stripe phases, as well as the spiral order. Our findings obtained in the Hubbard model within the SB approach for $t^{\prime}=-0.15 t$ agree qualitatively in the low doping regime $x \lesssim 1 / 8$ with the experimental data for the cuprates and reveal a strong influence of the next-nearest neighbor hopping $t^{\prime}$ on the optimal filling of DWs. Simultaneously, finite $t^{\prime}<0$ promotes the onset of the diagonal spiral phases and increases the optimal spiral pitch $\epsilon$. We therefore conclude that a large value of $\left|t^{\prime}\right|$ might be the reason why the static charge order has been detected in YBCO only in the highly underdoped regime [38]. 
MR acknowledges the hospitality of the Laboratoire CRISMAT where part of this work has been done. This work was supported by the Polish Ministry of Science and Education under Project No. 1 P03B 068 26, and by the Ministère Français des Affaires Etrangères under POLONIUM 09294VH.

\section{REFERENCES}

[1] Maekawa S. et al., Physics of Transition Metal Oxides (Springer Verlag, Berlin) 2004.

[2] Lee P. A., Nagaosa N. and Wen X.-G., Rev. Mod. Phys., 78 (2006) 17.

[3] V. Hinkov et al., Nature (London), 430 (2004) 650; V. Hinkov et al., arXiv:cond-mat/0601048 (unpublished).

[4] Seibold G. and Lorenzana J., Phys. Rev. Lett., 94 (2005) 107006; ibid. Phys. Rev. B, 73 (2006) 144515; Vojta M., Vojta T. and Kaul R. K., arXiv:cond-mat/0510448 (unpublished).

[5] Kivelson S. A. et al., Rev. Mod. Phys., 75 (2003) 1201.

[6] Tranquada J. M. et al., Nature, 375 (1995) 561.

[7] Abbamonte P. et al., Nature Physics, 1 (2005) 155.

[8] Yamada K. et al., Phys Rev. B, 57 (1998) 6165.

[9] Dai P., Mook H. A., Hunt R. D. and DoĞan F., Phys. Rev. B, 63 (2001) 054525.

[10] Damascelli A., Hussain Z. and Shen Z.-X., Rev. Mod. Phys., 75 (2003) 473.

[11] Wróbel P. and Eder R., Phys. Rev. B, 62 (2000) 4048.

[12] Zacher M. G., Eder R., Arrigoni E. and Hanke W., Phys. Rev. Lett., 85 (2000) 2585.

[13] Fleck M., Lichtenstein A. I., Pavarini E. and Oleś A. M., Phys. Rev. Lett., 84 (2000) 4962; Fleck M., Lichtenstein A. I. and Oleś A. M., Phys. Rev. B, 64 (2001) 134528.

[14] Wróbel P., Maciąg A. and Eder R., arXiv:cond-mat/0408703 (unpublished).

[15] Shraiman B. I. and Siggia E. D., Phys. Rev. Lett., 62 (1989) 1564.

[16] Hasselmann N., Castro Neto A. H. and Morais Smith C., Phys. Rev. B, 69 (2004) 014424.

[17] Lindgåd P.-A., Phys. Rev. Lett., 95 (2005) 217001.

[18] Giamarchi T. and Lhuillier C., Phys. Rev. B, 42 (1990) 10641.

[19] Tohyama T., Phys Rev. B, 70 (2004) 174517.

[20] Feiner L. F., Jefferson J. H. and Raimondi R., Phys. Rev. Lett., 76 (1996) 4939; Prelovšek P. and Ramšak A., Phys Rev. B, 72 (2005) 012510.

[21] Sнiн C. T. et al., Phys. Rev. Lett., 92 (2004) 227002.

[22] Frésard R. and Wölfle P., Int. J. Mod. Phys. B, 6 (1992) 685.

[23] Zimmermann W., Frésard R. and Wölfle P., Phys. Rev. B, 56 (1997) 10097.

[24] Raczkowski M., Frésard R. and Oleś A. M., Phys Rev. B, 73 (2006) 174525.

[25] Jefferson J. H., Eskes H. and Feiner L. F., Phys Rev. B, 45 (1992) 7959.

[26] Pavarini E. et al., Phys. Rev. Lett., 87 (2001) 047003.

[27] Frésard R., Dzierzawa M. and Wölfle P., Europhys. Lett., 15 (1991) 325.

[28] Deeg M. and Fehske H., Phys. Rev. B, 50 (1994) 17874.

[29] Fano G., Ortolani F. and Parola A., Phys. Rev. B, 42 (1990) 6877.

[30] White S. R. and Scalapino D. J., Phys. Rev. B, 60 (1999) 753.

[31] Normand B. and Kampf A. P., Phys. Rev. B, 65 (2001) 020509.

[32] Wróbel P., Maciąg A. and Eder R., J. Phys.: Condens. Matter, 18 (2006) 1249.

[33] Seibold G. and Lorenzana J., Phys. Rev. B, 69 (2004) 134513.

[34] Lorenzana J. and Seibold G., Phys. Rev. Lett., 89 (2002) 136401.

[35] Ino A. et al., Phys. Rev. Lett., 79 (1997) 2101.

[36] Duffy D. and Moreo A., Phys. Rev. B, 52 (1995) 15607.

[37] Fleck M., Lichtenstein A. I, Oles A. M. and Hedin L., Phys. Rev. B, 60 (1999) 5224.

[38] Mook H. A., Dai P. and DoĞAn F., Phys. Rev. Lett., 88 (2002) 097004. 